\title{
LA INVESTIGACIÓN EVALUATIVA DE PROGRAMAS EDUCATIVOS CON TECNOLOGÍAS DE LA INFORMACIÓN Y LA COMUNICACIÓN: PROPUESTA DEL ESTADO DE LA CUESTIÓN**
}

\author{
José Miguel Rodríguez García
}

Recibido 26-V-2003 • Aceptado 12-VIII-2003

\begin{abstract}
Resumen: En este artículo se presenta una propuesta de estado del arte sobre investigación y evaluación de programas educativos que tiene como característica estar mediados por tecnologías de la información y la comunicación (TIC). En el artículo se revisan las principales intenciones y modelos de los enfoques evaluativos de tales programas, las tendencias generales en lo que respecta a la evaluación de impacto, las tendencias en el uso de indicadores y marcos de evaluación, los principales métodos y técnicas de evaluación, y finalmente el abordaje del tema de la equidad social en la evaluación educativa. Con estos elementos se realiza un balance que permite proponer una lectura del estado actual en esta novel temática.
\end{abstract}

Palabras clave: Programas Educativos, Mediación Tecnológica, Evaluación de Programas, Estado de la Cuestión, Investigación, Indicadores, Técnicas de Evaluación, Métodos de Evaluación.

\section{Introducción}

La discusión sobre la valoración de las repercusiones de la introducción de la informática a programas educativos se puede ubicar desde los comienzos mismos de estos programas en Europa, Norteamérica y Latinoamérica (Crook, 1998; Plaz, 1999). Luego de diversos avances en esta dirección el tema de la evaluación de tales programas sigue siendo uno de los más relevantes, y sobre el cual se dirigen múltiples esfuerzos en la actualidad (por ejemplo véase CAIBI, 2000 y 2001; Prat, 2001).

Es importante para toda investigación que se dirija a valorar los resultados, efectos o impactos de programas educativos que introduzcan el uso de Tecnologías de la Información y la Comunicación ${ }^{1}$ tener una visión general de cómo la investigación evaluativa ha sido planteada en este campo. Sin embargo, dado el extenso volumen y la diversidad de la información existente sobre la temática, se prefiere hablar de una propuesta del estado del arte; puesto que lo que se ofrece a continuación es una posibilidad entre otras, de sintetizar el estado de la cuestión en este campo del conocimiento.

El desarrollo del documento se estructura en seis partes. En la primera se 
presentan las principales características de los documentos revisados. En la segunda se explicitan las intenciones y los enfoques evaluativos que han seguido las investigaciones y propuestas teóricas en esta temática. Las tendencias en la evaluación de impacto social se abordarán en la tercera parte y se complementa en la sección cuarta, con una revisión de indicadores y los marcos de evaluación empleados. En la quinta se plantean los principales métodos y técnicas de evaluación. En la sexta sección, siguiendo nuestro interés por presentar las relaciones entre las TIC en educación y la equidad, se expondrá la forma en que esta temática ha sido abordada por las investigaciones. Finalmente se presenta una síntesis de aspectos generales que se desprenden de esta propuesta del estado de la cuestión y sus repercusiones en la investigación evaluativa.

\section{Características de la revisión documental}

La construcción de este documento supone una revisión de modelos de evaluación de proyectos que integran TIC en educación y en otros ámbitos. Esta revisión se basa en el estudio de diversas publicaciones sobre el tema. Se incorporan antecedentes de metodologías y discusiones conceptuales sobre evaluación e investigación del impacto social. Ello sin embargo, implica una exploración de carácter general y flexible, abierta a la consideración de marcos o propuestas de evaluación de impacto de proyectos o programas que integren componentes tecnológicos, aún y cuando su ámbito de aplicación trascienda el educativo y se instaure en lo organizacional, lo comunal o el desarrollo sostenible en general.

Con respecto a las evaluaciones generales que no hacen explícito el componente educativo pero que, sin embargo, realizan estudios que incorporan el uso de TIC (Athanasou 1999; Díaz-Albertini
2001; UNRISD 1998; Menou, 1999a; Menou \& Potvin, 2000, Lanfranco \& Balson, 2000; Young, 1997; Miller \& Slater, 2000; Rohozinski, 1998; Hamelink, 1999; Gómez \& Hunt, 1999; Olivier 2000; Wood 1997; Camacho, 2000a, 2001a \& 2001c; Blau, 2001; ACP-PSRM, 2001; McConnell, 1998; Daly, 1999), se observa una tendencia a analizar instituciones, programas y organizaciones más que individuos particulares. En su mayoría, estos documentos conciben la evaluación de TIC como evaluación de la Internet, de los procesos e interacciones en ella, de las redes de trabajo y de los espacios virtuales.

En cuanto a los estudios que hacen referencia explícita al ámbito educativo, destacan por su particularidad, aquellos que se refieren a telecentros dado que éstos se enfocan en el área rural (Gómez y Hunt, 1999; Ernberg, 1998; Colin \& Walker, 2001; Hudson, 2001; Menou, 2001b), y aquellos documentos que refieren específicamente a la aplicación de tecnologías en el ámbito escolar. Estos últimos incluyen la evaluación de aspectos como el software (Britain \& Liber, 1999 y Honey, McMillan \& Carrig, 1999), las condiciones infraestructurales para la incorporación de las TIC en programas educativos (OECD, 2001; Lemke, Martín, Capella; 2001), las características de los ambientes de aprendizaje (FOD, 1995b), el papel de los docentes en la puesta en práctica de programas educativos apoyados en las TIC (Alvarez, Roman, Dobles, Umaña, Zúñiga, García, Potashnik y Rawlings, 1998) y de los procesos de capacitación de los docentes (FOD, 2000a).

\section{Intenciones y modelos de los enfoques evaluativos}

La reflexión en torno a los modelos subyacentes a los documentos sobre evaluación revisados, permite distinguir variados aportes conceptuales y metodológicos según los supuestos y las características del enfoque mismo de evaluación. 
Si bien casi todos los documentos parecen conservar el sentido de la palabra evaluación como elemento de juicio para la toma de decisiones (Athanasou, 1999; Jackson, 2001; Menou, 1999; Menou \& Potvin, 2000; Menou, 2001; Potvin, Lanfranco \& Balson 2000; Young, 1997; Wood 1997; ACP-PSRM, 2001); el modelo y la intencionalidad de esta evaluación, son distintos.

El primer gran rasgo identificable en cuanto a la intencionalidad específica de la evaluación, corresponde al interés por proveer a los diseñadores y administradores de proyectos, de un conjunto de elementos de juicio que permitan determinar si la forma en que se está conceptualizando el programa podría obtener el impacto buscado. En esta tendencia destaca la propuesta de Jackson (2001), cuya lógica apunta a evaluar la coherencia en el establecimiento de los problemas, objetivos, estrategias y actividades específicas del programa sujeto a valoración. En este enfoque, la consistencia en los diversos niveles de especificidad, la jerarquización y la priorización, son entonces los elementos claves para planear, diseñar, implementar y evaluar proyectos (Jackson, 2001).

Una posición similar presentan los documentos de consultores internacionales para la valoración del impacto de la introducción de las TIC en el ámbito organizacional; en donde la evaluación es concebida como el proceso de aplicación de un método, que determina el desempeño de un programa para la consecución de los resultados esperados, durante un período de tiempo determinado. Ello con la intencionalidad de apoyar la toma de decisiones y la resolución de problemas; siendo el matiz particular, el de valorar el éxito en función del aporte que el uso de las tecnologías hayan brindado para el cumplimiento de la misión organizacional y sus objetivos (Carver, 1999; Drucker, 1999; McClure \& Lopata, 1999; Advance Strategic Management Consultants, 1999; Camacho, 2000a, 2001a, 2001c).

Otro ejemplo de esta intencionalidad presente de los marcos de evaluación, es el caso expuesto Athanasou (1999) quien, en su propuesta de evaluación de programas educativos de capacitación para el comercio y la industria, apunta que el fin que busca la evaluación del aprendizaje asistido por computadora en el ámbito del comercio, es que mediante la valoración de los programas se puedan tomar decisiones con respecto a los mismos, que contribuyan a la vez con su mejoramiento.

Un caso particular es expuesto por ACP-PSRM (2001) quienes proponen una metodología para evaluar proyectos que implementan el uso de las TIC pero desde una perspectiva de género. En este modelo lo que se busca es valorar como ha sido tomado en cuenta el factor género en tales programas y cuáles han sido sus impactos en esta dirección. Resultados de esta perspectiva serían empleados tanto para el mejoramiento, la retroalimentación, la valoración y la planificación de otros programas.

Otra tendencia subyacente que es posible reconocer, remite a la intencionalidad comparativa al interior de los proyectos mismos como mecanismo de orientación para la toma de decisiones en el transcurso de las diversas fases de implementación. Esta es la situación en las propuestas metodológicas de Menou (1999a, 1999b) y Menou \& Potvin (2000) quienes enfatizan la necesidad de documentar la situación previa al inicio del proyecto como condición base o control que se contrasta con la situación en diversas fases y que permite, en un modelo longitudinal, el monitoreo y la evaluación de impacto. Cabe apuntar sin embargo, que tanto Menou (1999a, 2000, 2001a), como Potvin (2000), y Potvin, Lanfranco y Balson (2000) formulan estos prerrequisitos y lineamientos a partir de sus lecciones aprendidas en evaluaciones expost de impacto con proyectos que introducían componentes tecnológicos en diversos contextos. De estas experiencias rescatan además, como elementos primordiales las siguientes recomendaciones:

Necesidad de una estandarización conceptual y un marco de trabajo 
rural que permita integrar las diversas poblaciones de interés.

- Importancia de establecer dominios de impacto de tal forma que pueda trascender la unidireccionalidad que se ha impreso a la evaluación del flujo de cambio y se puede valorar el impacto en la modificación recíproca entre tecnología y sociedad.

Específicamente, de la evaluación del impacto social de proyectos que utilizan tecnologías digitales, y particularmente en el marco de los preparativos para el pilotaje de la OPA Online Proposal Appraisal-, IDRC (2000); Menou y Potvin sugieren la conveniencia de realizar distinciones en los hallazgos según éstos resultados inmediatos, efectos, consecuencias, grado de satisfacción o impacto. Son también ejemplos de monitoreos o evaluaciones concurrentes las propuestas de Wood (1997); UNRISD (1998) y Athanasou (1999), quienes respaldan la realización de evaluaciones en distintas etapas de los proyectos con el fin de darles seguimiento a los procesos, o bien valorar el cumplimiento de tareas y la efectividad de las diversas fases entre otros.

La identificación de diversos dominios de impacto en los cuales se observen los hallazgos, parece ser otro rasgo común en las propuestas de los marcos de evaluación revisados y a ellos, se suman autores como Camacho (2000a \& 2000b); Miller \& Slater (2000) y Potvin, Lanfranco \& Balson (2000). Éstos últimos proponen un marco conceptual cuyo propósito es orientar estudios periódicos en proyectos longitudinales. Para ello, sistematizan categorías de uso de las TIC en donde podrían presentarse impactos, cuyos dominios son los diversos contextos comunitarios y las acciones posibles que son facilitadas por las TIC.

Un caso de aplicación que ilustra esta evaluación de impacto en dominios específicos, lo constituye el caso de la Red
Científica Peruana que evalúa cómo el uso de la red de Internet ha logrado cambios o efectos en la vida personal e institucional de los usuarios de la red en Perú (Díaz-Albertini, 2001). En este sentido, el autor afirma que "impacto" ha de entenderse en función de los cambios positivos o negativos que surgen a partir de la utilización de los servicios digitales al interior de los centros de trabajo, en la cotidianidad personal y en el entorno de estos individuos e instituciones.

Se reconoce así, en la evaluación de Impacto Social de la Red Científica Peruana, la intencionalidad de evaluar la efectividad de los resultados que se obtienen de la puesta en práctica de un proyecto en particular en, al menos, los siguientes dominios: usuarios, estructuras institucionales y calidad, cantidad, variedad de servicios y productos ofertados por los individuos y las organizaciones impactadas.

Subyace también en algunos de estos modelos la preocupación porque los resultados de la evaluación busquen el desarrollo de políticas que regulen el uso e incorporación de las TIC en los países (Díaz-Albertini 2001; UNRISD 1998; Hamelink, 1999; Sagna, 2000).

Otra tendencia identificable, refiere al concepto de progresión en los procesos evaluados que, conservando la categorización por dominios, supone una lógica en niveles ascendentes donde las condiciones de un nivel anterior, son pre-requisito para la evaluación en el nivel superior (Young, 1997). En este modelo, la intencionalidad es la de direccionar la toma de decisiones a partir de la evaluación gradual y continua en cada dominio, suponiendo condiciones mínimas en cada nivel como soporte de un impacto mayor en el siguiente.

Este marco de evaluación se sustenta en el reconocimiento de que las TIC apoyan el desarrollo sostenible de manera indirecta, como efecto secundario del apoyo directo que las mismas brindan para la coordinación y la información de individuos y grupos que trabajan a favor 
del desarrollo humano (Young, 1997). Específicamente, este marco de evaluación mide en el primer nivel los resultados directos de la introducción de las TIC a partir de las variables de información, conexiones sin límites, atemporalidad y mejora de costo-beneficios. Los resultados esperados son la formación de grupos y "clusters" en los que se genera conocimiento, cooperación, participación y acceso equitativo (segundo nivel) que, a su vez, generan nuevas formas de organización y cambios en las políticas (tercer nivel) que promueven el desarrollo sostenible con la creación de nuevos valores, prosperidad, salud y seguridad. Cada nivel además, supone la integración de dos componentes en la red de información: un componente físico (hardware) y otro humano e institucional.

Esta lógica progresiva posee además una implicación fundamental en la formulación de los hallazgos de las evaluaciones, categorizándolos según sean sus resultados (información sobre el progreso hacia el logro de objetivos en cada nivel); factores de desarrollo (cambios en diversos beneficiarios o poblaciones de interés) o factores administrativos. Cabe rescatar que la conveniencia de realizar distinciones en los hallazgos es sugerida también por Menou \& Potvin (2000).

En general, con respecto a los modelos de evaluación ya descritos, cabe apuntar que se observa la tendencia a la evaluación diagnóstica en distintos niveles (usuarios individuales, instituciones, organizaciones, comunidades, instancias privadas y gubernamentales) para ver el impacto social. Sin embargo, estos modelos tienden a buscar la descripción del estado de la cuestión de un fenómeno social que se reconoce cambiante y dinámico.

Otra área relevante para la investigación y la evaluación de impacto social gira en torno a los telecentros, que se caracterizan por ser un espacio físico que proporciona acceso público a las TIC para el desarrollo educacional, personal, social y económico (Gómez \& Hunt, 1999). Desde esta perspectiva, los telecentros han sido una estrategia privilegiada por parte de organizaciones gubernamentales y no gubernamentales para la promoción del desarrollo en diferentes puntos del mundo (Colin \& Walker, 2001).

$\mathrm{Al}$ respecto, se señalan varias limitaciones en el alcance de las evaluaciones, en tanto la mayoría de los recursos parecen haber sido destinados a la implementación de proyectos piloto que no tuvieron el soporte paralelo en el destino de recursos para la comprensión de sus usos e impactos en las comunidades.

Dos iniciativas relevantes en el intento de subsanar estas limitaciones son las propuestas de Ernberg (1998) y la de Hudson (2001), de una metodología que integre tanto un eje formativo como otro sumativo para la evaluación de proyectos de telecentros ${ }^{2}$. En ambas propuestas, el primero de estos ejes que se supone continuo, serviría de monitoreo para la identificación de problemas y oportunidades que retroalimenten la ejecución y promuevan nuevas estrategias de acción. El segundo, supone una evaluación expost que evalúe los logros, la relación costo-beneficio y el impacto del proyecto, de donde derivarían las lecciones aprendidas; estas últimas deberían constituirse en los insumos principales para la retroalimentación y futura planificación de programas.

Por otra parte, Hudson (2001) argumenta que una manera de consolidar las posibilidades de que un programa que actúa por medio de telecentros tenga un impacto real, es considerado desde la planificación, las características de la comunidad en tanto que género, etnia, nivel educativo, nivel de ingresos y localización geográfica; las cuales, además de consolidar la capacidad de impacto, introducen una perspectiva de equidad en el programa.

Por su parte Chasquinet (2002) propone enfocar la evaluación por medio del sistema $\mathrm{MEAI}^{3}$ que simultáneamente incorpora el monitoreo, la evaluación y el análisis de impactos en tres grandes escenarios 
constituidos por las instituciones promotoras, los telecentros y las comunidades; con el fin de valorar las consecuencias de los programas que implantan telecentros.

Para Menou (2001a) los telecentros constituyen medios adecuados para favorecer procesos de desarrollo social en las comunidades. Sin embargo estos procesos deben trascender al desarrollo de habilidades en las personas para la interacción con las TIC y no deben de permanecer en el nivel de la alfabetización digital. Además, la conectividad por si misma no es una garantía del desarrollo cuando se carece de personas capacitadas óptimamente para tener un desempeño adecuado en estas condiciones. Esta tesis también es sostenida por Young (1997) quien señala que para la generación de conocimiento se requiere no sólo de la disponibilidad de la información sino de la adecuada interacción de las personas.

Según la postura de Menou (2001b), los intentos de ligar el desarrollo social de comunidades, con el desarrollo de habilidades individuales para la interacción con las TIC, deben concretarse en el desarrollo de mecanismos de evaluación que den cuenta de estos procesos. Estos mecanismos son denominados como IsTICometros y se deben dirigir principalmente al desarrollo de indicadores económicos, indicadores de desarrollo humano y social e indicadores de información e infraestructura de las TIC.

Desde el punto de vista de las dificultades que se generan con la sinergia entre desarrollo de habilidades con la tecnología y desarrollo social, una de las fuerzas que puede impedir la interacción de las personas con la tecnología en el espacio de la comunidad, especialmente cuando esta es rural, es la "tecnofobia" que se puede desarrollar a propósito de las TIC. Ante este peligro una de la soluciones más interesantes es constituir las comunidades de aprendices (Wilmore, 2001) que es un método de trabajo en el que todos los miembros de la comunidad son integrados al proceso de aprendizaje por medio del trabajo en conjunto, la investigación y la búsqueda de soluciones colaborativas.

Gómez \& Hunt (1999) afirman sin embargo, que mientras no se desarrollen metodologías y herramientas apropiadas para evaluar adecuadamente el impacto social del uso de las TIC en actividades de desarrollo, la credibilidad de los proyectos que implementen el uso de TIC seguirá estando basada en la euforia dominante que pretende ver en la tecnología la solución a los problemas sociales.

En síntesis, las evaluaciones e investigaciones en programas que introducen TIC han sido direccionados a valorar resultados, efectos e impactos con el fin de obtener insumos para la toma de decisiones, ya sean estos con el fin de elaborar diseños apropiados de programas que serán ejecutados en el futuro, monitorear y retroalimentar programas en ejecución o valorar las consecuencias concretas de los mismos.

En cuanto a los modelos específicos, resaltan aquellos que se dirigen a construir evaluaciones por niveles, donde cada uno de ellos constituyen elementos necesarios para alcanzar otros de mayor alcance o complejidad en sus consecuencias. Esta perspectiva es válida tanto en el caso de los telecentros como en el caso de otros programas mediados por TIC. Sin embargo, se puede concluir que a pesar de la experiencia en investigación evaluativa en esta temática, existe una tendencia a reflexionar sobre la escasez de modelos adecuados para valorar el impacto de tales programas lo cual se traduce en la carencia de un instrumental.

\section{Tendencias generales en la evaluación de impacto}

En la evaluación de impacto social y las condiciones de equidad de los programas que introducen nuevas tecnologías de la información y la comunicación en el 
ámbito educativo, destacan los aportes de Britain \& Liber (1999), SchoolNet (2000), proyecto Conexiones (2001), Honey, McMillan \& Carrig (1999) y Herman (2001).

Los primeros, innovan al señalar que las estrategias de evaluación de programas educativos con TIC, (en su caso, la evaluación de ambientes de aprendizaje virtual), no han de buscar la discriminación en nociones de calidad; en tanto lo fundamental es la generación de un marco que permita revelar las suposiciones pedagógicas que subyacen al software mismo, o a la práctica con la que es introducido en el sistema educativo, ya que de ello depende su impacto.

Los segundos plantean la necesidad de evaluar la práctica en el aula misma con un enfoque formativo dirigido a los docentes de los programas de desarrollo educativo que introducen TIC. Sugieren, a diferencia de Britain \& Liber (1999) que la evaluación de impacto ha de explorarse en la calidad de la enseñanza y el aprendizaje, y que ello toma lugar en cuatro niveles distintos, a saber, el nivel de las habilidades para el uso de las TIC: el nivel de la integración a partir de la evaluación de competencias; el nivel de crecimiento profesional de los educadores y el nivel de los cambios en la escuela en general. Planteamientos semejantes se encuentran en Alvarez y otros, 1998. Este estudio se propuso analizar comparativamente la puesta en práctica de los programas de informática educativa para primaria de Costa Rica, contemplando tres niveles de análisis: el programa, la escuela y el aula, prestando especial atención al papel de los docentes en el funcionamiento de los programas en esos tres niveles.

Honey, McMillan \& Carrig (1999) presentan una discusión en torno a lo aprendido durante las tres décadas de investigación sobre tecnologías educativas en el Centro EDC'S para la Infancia y la Tecnología en Estados Unidos. En ella, se sugiere que la revisión ha develado tres grandes factores que impulsan la investigación en este campo hacia nuevas áreas de exploración, a saber, los cambios en la naturaleza de los elementos tecnológicos; los cambios en los tipos de preguntas de investigación que se hacen y los cambios en las formas y métodos de investigación que se utilizan.

$\mathrm{Al}$ respecto, señalan que los estudios durante los años 70 y los 80 lograron demostrar el impacto de las tecnologías o el software sobre el aprendizaje de los estudiantes, pero que éstos, sin embargo, eran íntimamente dependientes del tipo particular de tecnologías que estaban siendo utilizadas por los sujetos del estudio. Apuntan adicionalmente que, como estos estudios se enfocaban específicamente en ciertas tecnologías y en su impacto particular, contribuyeron muy poco con el objetivo de investigar para discernir los roles generalizables que las tecnologías pueden jugar en la consecución de retos claves en el proceso de enseñanza-aprendizaje (Honey, McMillan \& Carrig, 1999).

Estos autores anotan que por ello, y por la combinación de la computación, la conectividad, las capacidades visuales y multimediales, la miniaturización y la velocidad en los actuales elementos tecnológicos, es que se introducen cambios en la esfera de los problemas de investigación que se han de plantear. Las preguntas inicialmente formuladas en los años 70's buscaban encontrar cómo cierto tipo de actividades mediadas por la computadora mejoraban el aprendizaje del estudiante, siendo un problema fundamental de este tipo de planteamientos, la ausencia de preguntas por el proceso mayor de cambio a escala institucional y el contexto social en el cual eran utilizadas y adquirían sentido. Las prácticas de enseñanza, las experiencias del estudiantado, los contextos pedagógicos e inclusive lo que estaba siendo realizado con las computadoras, fueron excluidos para que los investigadores pudieran hacer afirmaciones poderosas y definitivas sobre los efectos de la introducción misma de la tecnología. Este camino probó 
su esterilidad para la investigación del impacto de las TIC en los procesos educativos.

El Proyecto Conexiones visualizó este problema en su documento "Modelo de Evaluación" (2001), al señalar que el campo de la incorporación de nuevas tecnologías a la educación se observa una tendencia a realizar evaluaciones e investigaciones sobre tópicos específicos, con un enfoque que intenta identificar y explicar la influencia de un factor determinado en un resultado concreto. Subrayan los autores que ello supone una gran limitación, en tanto los hallazgos no permitían a los educadores alcanzar el gran reto de utilizar esas tecnologías para apoyar el desarrollo de capacidades en los estudiantes.

Surgen entonces nuevas preguntas de investigación que rescatan interrogantes como las siguientes: ¿cómo se integran las tecnologías en los contextos educacionales?, ¿cómo son interpretados y adaptados por los usuarios los nuevos recursos electrónicos? y ¿cómo articular las nuevas capacidades tecnológicas con las necesidades de aprendizaje de los estudiantes? y ¿cómo puede interactuar y apoyar el cambio tecnológico con los cambios en otras esferas del proceso educativo? A estas preguntas se les puede agregar aquellas que elabora Jurich (1999) en su cuestionamiento de algunas de las evaluaciones realizadas a estos programas: ¿cómo se integra la informática al currículo escolar en general?, ¿cuáles son los factores culturales que median en el proceso de aprendizaje tanto en el docente como en el estudiante? No obstante, este enfoque más contextual de la investigación del impacto de Programas Educativos apoyados en las TIC se encuentra planteado en la metodología de los primeros estudios conducidos por la Fundación Omar Dengo (1992, 1995).

Estas nuevas preguntas, afirman Honey, McMillan \& Carrig (1999), requieren del desarrollo de métodos que puedan responder a cómo los factores de diseño, cultura escolar y prácticas educativas y pedagógicas dan forma al impacto que las tecnologías pueden tener en el sistema escolar. Para ello recomiendan la realización de estudios longitudinales de gran escala que busquen entender cómo sucede el proceso de innovación en las escuelas y no se limite a encontrar los resultados específicos. Igualmente subrayan la importancia de rescatar el rol fundamental de los maestros como colaboradores en el diseño, la implementación y la interpretación de la evaluación.

Respondiendo a esta inquietud, Van Harmelen (2001) diferencia entre evaluaciones de impacto y el "impact assessment". La evaluación debe tomar en cuenta las influencias positivas y negativas que no son necesariamente atribuibles al proyecto específico; mientras que, "assessment" describe un proceso sobre el impacto que la intervención tiene y está acorde con la naturaleza cualitativa del estudio.

Esta tesis es sostenida también por Crook (1998) quien en el análisis de resultados de investigaciones dirigidas a la evaluación de efectos producidos por la introducción de la informática en el sistema educativo en los 80's y 90's, llega a la conclusión que la variable que demuestra tener un peso significativo en el aprovechamiento de los alumnos se encuentra en la capacitación de los docentes y la implementación de estrategias pedagógicas adecuadas al medio. También Herman (2001) hace hincapié en esta idea demostrando que la reforma educativa no se sostiene únicamente con la introducción de la tecnología sino que se debe de enfatizar en la puesta en práctica de tales programas. Este es el aspecto crucial a tomar en cuenta en las evaluaciones.

Específicamente en el ámbito latinoamericano, un aporte fundamental en materia de evaluación proviene del Proyecto Conexiones en Colombia anteriormente mencionado. Según afirman, el enfoque que se sigue en dicho proyecto reconoce que la evaluación permite comprender la naturaleza del programa, con la intención de mejorar a través del análisis y 
de los resultados la práctica misma. $\mathrm{Al}$ respecto añaden que la evaluación que se propone no tiene solamente la pretensión de etiquetar el programa como bueno / malo, ni siquiera la de facilitar la toma racional de las decisiones, sino que convierte en un instrumento que coloca el énfasis en los procesos y el contexto de los ambientes de aprendizaje más allá de la medición de los productos, las condiciones de entrada y los recursos mismos (Proyecto Conexiones, 2001).

En este sentido, es oportuno también el señalamiento que hace Aphek (2001) en torno al concepto de aproximación dialógica en la metodología de evaluación en sistemas educativos. Al respecto rescata que existe un proceso de diálogo permanente entre componentes tales como tópicos, flujos de información, habilidades y valoración de la comunidad específica en la cual opera la escuela y programa.

A pesar de lo anterior, las investigaciones y evaluaciones focalizadas en aspectos particulares no han sido dejadas de lado y por el contrario son tan relevantes como en los primeros años del desarrollo de esta temática. Un ejemplo de ello se puede observar en el estudio de Allegra, Chifiari \& Ottaviano (2001) en la que se afirma la relevancia de las TIC en el desarrollo cognoscitivo a partir de resultados favorables en aspectos como la creatividad y el uso del tiempo.

En general, estas evaluaciones e investigaciones en el campo de la informática educativa, sugieren que el acceso sostenido a la tecnología tiene el potencial de impactar positivamente tanto en el proceso de aprendizaje de los estudiantes como la visión de la comunidad sobre las capacidades de sus estudiantes. Sin embargo, los hallazgos indican que la tecnología por y en sí misma, con la ausencia de otros componentes de reforma escolar, no producen este tipo de cambios (Honey, McMillan \& Carrig, 1999). Una idea similar es ejemplificada por la propuesta de una concepción relacional de la tecnología (Burbules
$\&$ Callister, 2001) que reconoce que las elecciones en materia de uso de las TIC siempre están relacionadas con un cúmulo de otras prácticas y procesos sociales que determinan su impacto.

Lo anterior también se evidencia en planteamientos como los de Hamelink (UNRISD, 1999) e Iglesias (BID, 2000), quienes afirman que el progreso y el desarrollo social requieren de una plataforma de espacios públicos, redes, canales y sitios que faciliten el intercambio de conocimientos para la deliberación, participación e información de las personas con miras a la democratización de la toma de decisiones en las estructuras públicas locales, naciones y globales. Así, se reconoce el potencial de las TIC para desarrollar esta plataforma, pero nunca por sí mismas, sino enmarcadas en políticas que orienten su uso. En este sentido Iglesias (2000) afirma que la voluntad política tiene el potencial de marcar la diferencia entre brecha digital y oportunidad digital para el desarrollo, y que las tecnologías de la información y la comunicación, sin dicha voluntad política para el logro de objetivos de desarrollo, puede más bien incrementar la brecha ya existente en la sociedad del conocimiento.

En síntesis, las tendencias en la evaluación del impacto social plantean la necesidad de no colocar a la tecnología en el centro del análisis. Por el contrario la tecnología debe verse como una variable más en un conjunto de otras variables que tienen un enorme peso en las consecuencias finales de los programas que se sirven de las TIC. Por otra parte, las evaluaciones pueden estar ubicadas en el orden de lo general cuando tienen la ambición de valorar la generalidad de los impactos de los programas; pero también se pueden centrar en aspectos específicos de los efectos. Estos últimos a pesar de que no tienen la posibilidad de hacer una completa valoración de impacto social sí muestran datos importantes para evaluaciones más generales. 


\section{Tendencias en el uso de indicadores y marcos de evaluación}

Los diferentes documentos revisados para esta propuesta de estado del arte, pero, especialmente aquellos que constituyen evaluaciones o reflexiones metodológicas para la evaluación de impactos de TIC, presentan como una de las labores esenciales en la construcción de sus marcos metodológicos la elaboración de indicadores pertinentes para la valoración del fenómeno de interés. Esta preocupación se observa en dos aspectos; por un lado, la construcción y escogencia de los indicadores en sí mismos, y por el otro, la construcción de estructuras de evaluación desde las cuales dichos indicadores deben ser empleados.

En la construcción de indicadores, se parte del supuesto de que los indicadores para valorar el impacto de las TIC poseen condiciones particulares, y en consecuencia, deben ser adecuados para que den cuenta del proceso que se quiere evaluar (Rohozinski, 1998). Sin embargo, este no parece ser un punto ampliamente compartido. Específicamente en el campo de la investigación y evaluación educativa, se encuentran documentos (Ritcher, 2001; Martinello, 1999) que hacen caso omiso de este punto de partida y buscan valorar el impacto de las TIC en términos de indicadores ampliamente usados en el pasado (por ejemplo: tasa de matriculación, rendimiento académico, deserción, etc.). Quienes presentan propuestas que buscan rescatar la particularidad de las TIC incluyen indicadores que se relacionan, por un lado, con las características de la interacción entre docentes y educandos con y a través de la tecnología (por ejemplo: uso, apropiación, desarrollo de ambientes colaborativos, etc.); y por otro, indicadores que valoran las consecuencias de las TIC más allá de los ámbitos inmediatos de su aplicación (por ejemplo: repercusiones de las TIC sobre el desarrollo social, generación de integración social, mejoría en condiciones de vida, etc.).
Con respecto a las estructuras de evaluación o marcos metodológicos que coordinan el empleo de indicadores, se perfilan al menos cuatro posiciones que se diferencian entre si con claridad y una quinta que constituye una posibilidad de integración.

La primera propuesta (Ernberg, 1998; National Research Council, 1998) que se puede encontrar es la de organizar las estructuras de evaluación según el ámbito de impacto. Estos niveles de impacto son de muy variadas posibilidades entre las que cabe señalar la escuela, el aula, la casa o la comunidad como un ámbito de impacto de las TIC. En general se refieren a ámbitos de impacto como espacios donde este impacto puede ser observado, la decisión de cuál es el ámbito escogido es una decisión que responden a los interese de la evaluación.

El segundo marco de organización empleado es entender los impactos según tipo de impacto. Se realizan organizaciones de indicadores según ubiquen resultados positivos o negativos, previstos 0 imprevistos, directos o indirectos (ver: National Research Council, 1998).

El tercer marco de ordenamiento de los indicadores responde al criterio de las condiciones específicas y las características de las poblaciones. En este sentido se ordenan marcos en función del tipo de población (Jackson, 2001; Young, 1997; Wood, 1997). Esto implica que el ordenamiento de los indicadores responde a criterios tales como genero, etnia, edad, nivel educativo, nivel económico, etc.

Un cuarto criterio de ordenamiento propone estructurar los indicadores en función del proceso del programa. Emplear la dimensión procesal del impacto implica considerar diferenciaciones tales como indicadores de penetración como antecedente a los indicadores de uso (Daly, 1999) o indicadores para resultados, efectos e impactos (Camacho, 2000b \& 2001a).

Finalmente, una visión integradora de los anteriores ordenamientos se puede 
observar en el trabajo de Proyecto Conexiones (2001) en el cual, el ordenamiento de su Modelo de evaluación comprende agrupar indicadores según el contexto (o ámbitos), condiciones, el proceso de programa y las poblaciones afectadas.

En síntesis se puede señalar que la construcción de indicadores para valorar las repercusiones de las TIC se orienta en función de diseñar indicadores que puedan dar cuenta de la especificidad del fenómeno. Esto, sin embargo no es ampliamente seguido homogéneamente y se mantiene el uso de los indicadores usados de forma tradicional en educación. Por su parte, los modelos de evaluación que les proveen a los indicadores de una estructura para su utilización, muestran variadas posibilidades. A pesar de ello, queda claro que estas diferentes posiciones no son de ninguna manera irreconciliables y por el contrario parece adecuado diseñar modelos de evaluación que integren dos o más de estas posibilidades.

\section{Principales métodos y técnicas de evaluación}

En cuanto a estrategias metodológicas, las propuestas de los documentos revisados son diversos. Sin embargo, hay consenso en cuanto a la necesidad de incluir a representantes de todas las poblaciones interesadas en el diseño, la implementación y el análisis del proceso de evaluación (Stoll, Menou, Camacho, Khellady, 2002; Menou, 1999 \& 2001; Menou \& Potvin, 2000; Potvin, Lanfranco \& Balson, 2000; Young, 1997; Jackson, 2001; ACP-PSRM, 2001). En este sentido, Athanasou (1999) plantea que cualquier programa o servicio de aprendizaje asistido por tecnología refleja una inversión tanto personal, como social y organizacional que necesita ser revisada.

Jackson (2001) postula la necesidad de que se lleven a cabo talleres de trabajo grupal con fases de análisis y planteamiento en los que recomienda integrar técnicas como la lluvia de ideas; la construcción de un árbol en los que se ramifican problemas u objetivos con una lógica subyacente de causa -efecto; la realización de FODA (Fortalezas, oportunidades, debilidades y amenazas); el análisis del campo de fuerzas positivas y negativas que promueven o inhiben la consecución de los objetivos del proyecto; y la creación de matrices que contengan objetivos, actividades, resultados esperados, posibles indicadores y factores externos que podrían afectar la consecución de los objetivos.

Esta necesidad de incorporar métodos grupales es también planteada por Menou (1999) quien introduce la recomendación de que en las diversas fases del proyecto, puedan verse reflejados aspectos de uso e impacto relacionados con las esferas de lo individual y lo grupal. De su propuesta puede extraerse una concepción de lo grupal, y el impacto en este nivel, como distinto a la suma de experiencias individuales por lo que, en la elección de los métodos específicos, esta condición merece el uso de grupos focales y grupos de discusión además de los cuestionarios y las entrevistas individuales. En este aspecto en particular, la propuesta de Menou (2001a $\& 2001 b)$ se dirige a apoyar evaluaciones con mecanismos que amplíen la participación de los interesados en virtud de que la valoración no debe centrarse en la visión de una elite dominante de actores en el proyecto.

Sumadas a estas recomendaciones, apunta como lecciones aprendidas de la segunda fase del programa "Impacto de la Información" en el marco del proyecto "Building on IDRC's Research Program on Assessing the Impact of Information on Decision Making". (Menou, 1999) la necesidad de clarificar, definir y documentar el concepto de impacto que se tomará como base, pues ello guía y determina los objetivos y procedimientos en las diferentes fases metodológicas de una evaluación. Retoma como métodos de investigación de impacto 
principalmente utilizados, los cuestionarios y las entrevistas semi-estructuradas; apuntando además, como se señaló previamente, la necesidad de que existan grupos controles en estudios longitudinales. Finalmente propone la pertinencia de producir un manual detallado que permita rescatar los marcos conceptuales y metodológicos en fases posteriores de un mismo estudio, para articular la pluralidad del uso de múltiples canales, fuentes y métodos de recolección de la información.

Por su parte, ACP-PSRM (2001) plantea que la identificación e incorporación de todos los interesados a diferentes niveles constituye una de las herramientas más poderosas en la evaluación TIC con perspectiva en género. Una de las razones para ello es que permite asegurar que se tomen en cuenta la perspectiva de grupos con diferentes características pero que igualmente están siendo afectados por las diferencias de género.

En este mismo sentido y en el ámbito educativo específicamente, Britain \& Liber (1999) rescatan la necesidad de considerar las diversas poblaciones implicadas: maestros, tutores, estudiantes e institución, y para ello consideran el modelo conversacional (Laurillard, 1993), que enfatiza las interacciones entre el estudiante y el tutor; y el modelo de sistemas viables (VSM, 1981; Beer, 1981) que subraya cómo el software ayuda al tutor a manejar conversaciones y construir actividades individualizadas para una gran número de estudiantes.

Miller \& Slater (2000) abogan por la pertinencia de los abordajes etnográficos del fenómeno suscitado en la incorporación de las TIC a las comunidades. Estos métodos de investigación dan cuenta de aspectos como los cambios en los procesos de socialización y apropiación, donde las TIC juegan un papel importante en la dinámica de los factores culturales. Aplicaciones concretas de este tipo de métodos de investigación se encuentra en el trabajo de Cabrera (2001) quien recurre a diferentes técnicas cualitativas con una perspectiva etnográfica para entender la apropiación de Internet en jóvenes escolares desde el punto de vista psicosocial y cultural. Por su parte el trabajo de Arredondo (2001) compara, con métodos etnográficos, las diferencias que existen entre las intenciones del proyecto Enlaces de Chile con los resultados. Por su parte, los estudios realizados en la FOD sobre el Programa de Informática Educativa para la escuela primaria, también han privilegiado los métodos cualitativos como los estudios de casos, basados en técnicas como la observación y la entrevista semiestructurada (FOD, 1993, 1995a, 1995b, 1997, 2000b). Finalmente, el trabajo de Van Harmelen (2001) asume un método naturalista/interpretativo que no se distancia de los enfoques etnográficos en la evaluación de un proyecto educativo con TIC en Namibia.

En general, los documentos revisados sugieren como métodos posibles además del trabajo con grupos focales y entrevistas, las observaciones, el estudio de casos, las aproximaciones etnográficas, el uso de cuestionarios por e-mail como mecanismos de recolección de información y los diseños experimentales (Hudson, 2001; Athanasou, 1999; Menou , 1999; Advance Strategic Management Consultants, 1999; Díaz-Albertini, 2001), el uso de técnicas participativas (Gómez \& Hunt, 1999; Sguazzin \& du Toit, 2000) y el análisis de anécdotas (Stoll, Menou, Camacho \& Khellady; 2002; PANTLEG, 2000).

Se rescata además la pertinencia de completar el trabajo de entrevistas con trabajo de campo, y en ello aparece como un rasgo fundamental la necesidad de integrar criterios y medidas objetivas o cuantitativas, en tanto factibles, medibles y verificables; y medidas subjetivas o cualitativas en tanto opiniones, percepciones y niveles de satisfacción. (Athanasou, 1999; Young, 1997; Jackson, 2001; Menou \& Potvin 2000; Advance Strategic Management Consultants, 1999; Wood, 1997; Hudson, 2001). A esta idea se suma Díaz-Albertini 
(2001), Miller \& Slater (2000) quienes rescatan que la medición no sólo hace referencia a los cambios tangibles o materiales sino también a las expectativas, percepciones y conocimientos de las personas.

\section{Abordajes de la equidad en el ámbito de las TIC}

La perspectiva de la equidad es una de las más relevantes en la discusión sobre las TIC. La incorporación de las TIC en los diferentes ámbitos de la vida social se ve principalmente como una necesidad más que un lujo, que permitirá el alcance de bienestar en el campo de la salud, la educación, y el empleo (Lawson \& Comber, 2000). Otros más optimistas aún, plantean que por medio de la incorporación de las TIC, la pobreza mundial puede disminuir hasta en un 50\% (Morrison, 2001); o en general las consideran herramientas altamente efectivas para promover el desarrollo social (Hilbert, 2001). En general se asume que la única forma de lograr avances sobre el tema de brecha digital es mediante la introducción de las TIC en la educación, pero ello depende de políticas sociales en las que tanto los gobiernos como las instituciones privadas participen activamente (Thomas, 1998).

A pesar de ello, existen diferentes discusiones sobre el tema de la brecha digital a nivel mundial (The Public Voice, 2001), especialmente en lo que se refiere a cuáles son las mejores políticas a seguir para eliminarla. Para ello la vía privilegiada ha sido la constitución de programas educativos de informática que presentan un acento en poblaciones tradicionalmente limitadas en posibilidades como son: las poblaciones rurales, el grupo femenino, la niñez y los grupos con bajos niveles económicos y educativos.

En este contexto el discurso prevaleciente es aquel que apunta a señalar el acceso físico a las TIC como un paso necesario pero no suficiente. En este sentido,
Kleiman (2000) expone que la equidad no debe ser buscada por medio de la disposición equitativa de máquinas entre alumnos, o entre comunidades con amplias posibilidades económicas y aquellas que carecen de ellas. La posición de acceso físico per se a las TIC responde a mitos generados por la inclusión de la tecnología en los sistemas educativos. Para que se dé la verdadera equidad debe de hacerse un uso de la máquina como herramienta. Esto implica que debe de darse un acento en la capacitación de los docentes y el desarrollo de estructuras organizativas que permitan la introducción tecnológica en las escuelas.

En esta línea de discusión, Mansell \& When (1998) presentan a las TIC como una posibilidad para que se alcance el "empowerment" de las personas. Un ejemplo de esto se puede encontrar en el estudio de Hafkin \& Taggart (2001) donde se expone como la introducción de TIC en los grupos de mujeres ha permitido que se den cambios en el proceso de producción, se han introducido nuevos productos y servicios y se ha dado un giro en la tecnología empleada, facilitando que las mujeres asiáticas eleven su nivel de vida. Un ejemplo específico del trabajo sobre la pobreza se encuentra en Aminata (2000), quien presenta el reporte de una experiencia en las que se comenta cómo se trabajó con jóvenes de Senegal para capacitarlos en el uso de tecnologías, esto con el fin de reducir la pobreza y proveer posibilidades para su futuro.

Desde el punto de vista de las zonas geográficas, la introducción de las TIC en las zonas rurales se considera una de las principales direcciones sobre las que se busca actuar contra la inequidad. Estas zonas tienen la particularidad de ser mayormente desprovistas de desarrollo social y aglutina la mayoría de la población mundial alcanzando un 71\% (PANTLEG, 2001). Esta perspectiva ha conducido a programas de informática educativa como el de Costa Rica a contar entre sus criterios para la selección de escuelas participantes, su ubicación en la zona rural y su 
pertenencia a los estratos sociales más desposeídos, de modo que aproximadamente el $60 \%$ de las escuelas que lo integran se ubican en la zona rural (FOD, 2002).

Para Ishaq (2001) a pesar de que existe brecha de la equidad en tanto que brecha económica y brecha en el acceso a la tecnología, lo más relevante es que por medio de estas se potencie la disminución de una brecha en la creatividad. Desde su perspectiva la Internet debe ser entendida como un espacio en el cual se puede desarrollar la creatividad de las personas.

La perspectiva de Morino Institute (2001) y al expuesta por Gómez \& Martínez (2001) es que la tecnología debe de ser empleada para el desarrollo de oportunidades, mediante las cuales se van a poder observar mejoras tangibles en las condiciones de vida de las personas. Este sería un objetivo que no se alcanzaría por la vía simplista de poner las TIC a disposición de las personas, sin que medie un enfoque orientado al desarrollo de las capacidades de las personas.

Estos últimos autores (Gómez, Martínez \& Reilly; 2001) plantean que la brecha digital es una manifestación de las brechas sociales, políticas y económicas, tanto en los ámbitos locales como internacionales. Por ello focalizarse en la brecha digital no va a ser determinante en la mejora del desarrollo social. Más que el acceso físico a la Internet y las TIC en general se requiere de la capacitación de las personas para que puedan emplearlas.

A manera de síntesis se puede señalar que existe en los documentos revisados una tendencia crítica a la sobrevaloración del efecto que las máquinas pueden tener en la sociedad y la educación. Esta sobrevaloración se traduce en políticas de distribución igualitaria de recursos tecnológicos; sin embargo, la idea de que la presencia de la máquina en sí misma produce desarrollo social y mejora las condiciones de equidad es un mito. Para alcanzar esto debe de partir de la perspectiva de la máquina como herramienta que es empleada para el desarrollo de capacidades humanas.

Por la vía del desarrollo de capacidades se puede avanzar hacia mejoras posibles en las condiciones de vida de las personas. Para que se dé esta mejora de las capacidades se requiere de inversión en la capacitación de docentes para que posibiliten un uso efectivo y apropiado de las TIC en las poblaciones interesadas.

Desde el punto de vista de la evaluación de programas que introducen las TIC en la educación, no se debe de limitar el análisis de la equidad al tema del acceso igualitario, sino que se debe avanzar hacia el conocimiento de efectos sobre las capacidades humanas y las condiciones para que ellas se desarrollen. Esto, claro esta, no excluye la preocupación por las variables de la desigualdad (género, pobreza, distribución geográfica, etc.); por el contrario busca pasar del análisis simplista de la presencia o no, a la valoración de cuál es la magnitud de los efectos y las condiciones que los posibilitan.

\section{Síntesis general}

Con el panorama anteriormente descrito se pueden extraer algunas conclusiones de orden general; las cuáles delinean -si bien es cierto, a grandes rasgos- intenciones, estrategias y énfasis en la investigación y evaluación de programas que emplean TIC. Estas líneas generales son la siguientes:

- La evaluación es entendida en el contexto de estos programas como un elemento para la formulación de juicios. Es frecuente el recurso a diferentes tipos de evaluación (exante, monitoreo, expost, etc.), y su empleo se debe principalmente a la intención con la que la evaluación se lleve a cabo; es decir, lo que realmente tiene peso es si la evaluación se realiza con miras a la planificación, la retroalimentación o a la 
toma de decisiones sobre el futuro de los programas. A pesar de ello, existe una tendencia a propiciar la confluencia de diferentes modalidades de evaluación en los nuevos modelos que se construyen para estos fines. Así, se busca complementar las evaluaciones diagnósticas con monitoreos y análisis de impactos, y de integrar evaluaciones sumativas y formativas.

- Se diseñan modelos de evaluación orientados a constatar los procesos de cambio producidos por la interacción de diversos contextos con las TIC, más que la búsqueda de resultados puntuales o lineales. Dado que la evaluación de los programas educativos que utilizan TIC suponen consecuencias progresivas y acumulativas, se plantea comprender estas consecuencias en función de modelos que den cuenta de procesos progresivos.

- Una situación particular que enfrenta la investigación y la evaluación de las consecuencias a nivel educativo y social de estos programas es el constante y acelerado desarrollo tecnológico, el cual le imprime cambios constantes a las posibilidades de aplicación de las TIC a la educación.

- Uno de los aspectos importantes que dificulta el desarrollo en metodología para la evaluación de los impactos sociales de los programas educativos apoyados en las TIC es la escasez de indicadores que puedan dar cuenta de los cambios que estos programas generan en los diferentes ámbitos en los que entran a interactuar.

- Existe bastante consenso en recomendar que con la instalación de un programa se debe de acumular la mayor cantidad posible de documentación, tanto de las condiciones iniciales como del desarrollo del programa.
Por esta vía se facilita la evaluación de impacto social y la evaluación longitudinal.

- Los metanálisis realizados a las investigaciones y evaluaciones desde los 70 sobre las consecuencias de las TIC presentan un panorama incierto respecto a la efectividad de estos programas, señalándose que son justamente las variables no tecnológicas (docencia, capacitación, relaciones interpersonales, etc.), las que tienen mayores y mejores efectos en el aprendizaje de las personas.

- En cuanto a métodos de investigación resalta el hecho de que el desarrollo de las evaluaciones debe darse con la incorporación de la mayor cantidad de representantes posibles de los interesados. En este sentido se consideran poco recomendables los enfoques en los que todo el protagonismo del proceso lo lleve a cabo el evaluador. Por otro lado, introducir a los diferentes grupos de interesados implica pensar los efectos de los programas apoyados en las TIC más allá de los ámbitos en los que tienen una incidencia directa. En este sentido, ámbitos que otrora han sido de escaso interés como el macrosocial o el comunal -en los proyectos que no son telecentros- se presentan ahora como sujetos importantes de evaluación.

- Los métodos longitudinales surgen como uno de los métodos más recomendados pero a la vez, menos empleados. Se sostiene que dichos métodos podrían dar resultados más sólidos con los cuales poder valorar las consecuencias reales que se le pueden atribuir a dichos programas.

- $\quad$ Por otro lado, el uso de métodos etnográficos y de técnicas cualitativas se postula como una de estas estrategias 
metodológicas más empleadas en la actualidad. Esto contrasta con el uso de métodos de medición de carácter cuantitativo que no parecieran estar en el primer plano de interés de los investigadores en el campo.

- La preocupación por la equidad se ha presentado de dos maneras: por un lado en función de grupos marginados (mujeres, pobres, niños, etc.), pero también se ha organizado la discusión en función de diferentes condiciones de equidad; donde resalta la comprensión de la equidad como igualdad de bienes (computadoras, conexión, capacitación) y la equidad como desarrollo de oportunidades. Esta última noción de equidad es la más valorada, y se plantea como perspectiva que debe estar presente desde la planificación de los programas hasta su evaluación.

Finalmente, más allá de aspectos que pueden centrarse en la características generales de la evaluación de programas, se ha señalado que la construcción específica de condiciones para evaluación del impacto social de programas apoyados en las TIC es aún un proyecto por constituirse. Se plantea que no existe a la fecha un consenso sobre cuáles son los indicadores adecuados para valorar el fenómeno y sus vías de aprehensión. Por ello, la exploración de modelos de evaluación, el desarrollo de indicadores y criterios se convierte en un punto capital de la agenda de investigación de estos temas.

\section{Notas}

* Este trabajo se llevó a cabo con la ayuda de fondos asignados por el Centro Internacional de Investigaciones para el Desarrollo de Ottawa, Canadá (CIID-IDRC) y se desarrolló en el departamento de Investigación de la Fundación Omar Dengo. Para la redacción inicial de las primeras secciones de este documento se contó con la colaboración de la Bach. Mónica Salazar
Villanea. Para la revisión de esta versión se contó con la colaboración de Magaly Zúñiga.

1. En adelante, para referirse a las Tecnologías de la Información y la Comunicación se emplearán las siglas TIC.

2. Son programas que vinculan los recursos tecnológicos con las comunidades. Tienen diversas formas de implementarse, ya sea por medio de instituciones (municipalidades, escuelas, etc.) o por medio de estructuras movibles que se colocan en las comunidades. Si bien su orientación puede ser educativa, la mayoría de estos programas están centrados en el desarrollo social de las zonas en las que operan.

3. MEAI: Monitoreo, evaluación y análisis de impactos.

\section{Referencias bibliográficas}

ACP-PSRM. "Metodología de evaluación de género (MEG)". En http://www.apcwomen.org/gem/ Consultado 6/1/02. 2001.

Aminata, T. "Linking computers and community organizations in Senegal". www.idrc.ca/red_article_english.cf m?article_num $=719$ Consultado el 1/11/2001. 2000.

Allegra, M.; Chifiari, A.; Ottaviano; S. "ICT to tain students towards creative thinking". En:

http://ifets.ieee.org/periodical/ vol_2_2001/allegra.html Consultado 15/1/02. 2001.

Álvarez, M.; Roman. F.; Dobles, M.; Umaña, J.; Zúñiga, M.; García, J.; Means, B.; Potashnik, M.; Rawlins, L. Computadoras en las escuelas: estudio cualitativo de Chile y Costa Rica. E\&T: Education and Technology Series: Special Issue. 1998.

Aphek, E. "Sharing Knowledge and bridging gaps: children teaching children computer skills". Israel. En: 
http://www.iicd.org/base/

story_search_read?id=27

Consultado 15/1/02. 2001.

Arredondo, J. "Nuevas Tecnologías, educación y equidad. Un estudio etnográfico en las escuelas de Chile. FONDECYTChile. FLACSO-Ecuador. 2001.

Athanasou, J. "A framework for evaluating the effectiveness of technologyassisted learning". En: Virtual University Journal, Vol. 02, № 1, pp. 13-21.

http://www.emerald-library. com/ pdfs/50802aa2.pdf

Consultado el 17/05/01. 1999.

Blau, A. "More than Bit Players: how information technology will change the ways Nonprofits an Foundations work and thieve in the information age”. Nueva York: Surdna Foundation. En:

http://www.surdna.org/documents/ morefinal.pdf

Consultado el 8/1/02. 2001.

Britain, S.; Oleg, L. "A Framework for Pedagogical Evaluation of Virtual Learning Environments". University of Wales, Bangor. En:

http://www.jtap.ac.uk/reports/htm/ jtap-041.html

Consultado el 28/05/01. 1999.

Burbules, N.: Callister, T. "Educación, riesgos y promesas de las nuevas tecnologías de la información". Ediciones Juan Granica: Barcelona. 2001.

Cabrera, J. "Náufragos y navegantes en territorios hipermediales: Experiencias psicosociales y prácticas culturales en la apropiación de Internet en jóvenes escolares". Informe presentado a concurso Investigación sobre Impactos Sociales de las TIC en Latinoamérica y el Caribe. FLACSO, Sede Ecuador. 2001.
CAIBI "Relación de indicadores TIC en los países de la CAIBI". En: http://www. map.es/csi/caibi/ indicadores_lista._pdf Consultado el 7/1/02. 2000.

CAIBI “Que es CAIBI?”. En: www.map. es/csi/caibi/general/ o_2.htm

Consultado 1/10/01. 2001.

Camacho, K. (coord.) "Building an ICT evaluation framework. IDRC-Workshop. Memoria de los talleres realizados en Costa Rica. 13-15/12/2000. Material Mimeografiado. 2000a.

Camacho, K. "Marco de referencia de la investigación. ¿Cómo nos acercamos a la valoración de impacto de la Internet en las Organizaciones de la sociedad civil de Centroamérica? Fundación ACCESO: Costa Rica. 2000b.

Camacho, K. "La Internet, un gran desafío para las organizaciones de la sociedad civil centroamericana". Primer análisis de una encuesta regional. Fundación ACCESO. En: http://www.acceso.or.cr/publica/ grandesafio.shtml Consultado 07/11/01. 2001a.

Camacho, K. "Internet, ¿una herramienta para el cambio? Elementos para una discusión necesaria”. Fundación ACCESO. En:

http://www.acceso.or.cr/publica/ telecom/conocimiento22.shtml Consultado el 07/11/01. 2001b.

Camacho, K. “¿Cómo nos acercamos a la valoración del impacto de la Internet en las Organizaciones de la Sociedad Civil de Centroamérica?". Fundación ACCESO. En: http://www.acceso.or.cr/publica/ telecom/Framework.shtml Consultado 07/11/01. 2001c. 
Chasquinet "Estado del arte de los telecentros en América Latina y el Caribe". Ecuador. En:

http://chasquinet.org/pub/docs/

telelac/estarte.doc

Consultado el 13/2/02. 2002.

Colin, L; Walker, D. (ed.) "Telecentres: Case studies and key Issues". Vancouver: the Common wealth of learning". En:

http://col.org/telecentres

Consultado 7/1/02. 2001.

Crook, C. "Ordenadores y aprendizaje colaborativo". Madrid: Morata. 1998.

Daly, J. (1999) “A conceptual framework for the study of impacts of the internet. University of Maryland.

http:// www.bsos.umd.edu/ cidcm/papers/jdaly/concept.htm Consultado 14/6/01.

Díaz-Albertini, J. "Evaluación de Impacto Social de la Red Científica Peruana e Internet en el Perú 1991-1995”. Dirección Universitaria Coordinadora de Investigación Científica, Universidad de Lima. Internet, en:

http: //www.rcp.net.pe/VFORO/

memorias/ esp/diaz5.htm

Consultado el 29/5/01. 2001.

Ernberg, J. "Integrated Rural Development and Universal Access-Towards a Framework for Evaluation of Multipurpose Community Telecentre Pilot Projects Implemented by ITU and its Partners. Partnership and Participation in Telecommunications for Rural Development: Exploring What Works and Why", Conference Guelph University, Canada. En:

http://www.itu.int/

ITU-D-UniversalAccess/johan/

papers/guelph.htm

Consultado el 28/6/01. 1998.
Fundación Omar Dengo . "Mi experiencia con el programa de informática educativa MEP-FOD: Impacto del programa en una escuela de zona rural". Informe 1-93. Informe de investigación evaluativa. San José. 1993.

Fundación Omar Dengo. "Vivencia en el laboratorio de informática educativa del PIE MEP-FOD. Un acercamiento a los procesos generados en maestras y encargadas de laboratorio". Informe de investigación evaluativa. San José. 1995a.

Fundación Omar Dengo. "La vivencia en el laboratorio de informática educativa del PIE: un acercamiento a los procesos generados en niños y niñas". Informe 2-95. Informe de investigación evaluativa. San José. 1995b.

Fundación Omar Dengo. "Construcción de lecciones aprendidas sobre la puesta en práctica del Programa de Informática Educativa MEP-FOD: el papel de los docentes". Informe \#6, Lecciones Aprendidas. Informe de investigación. San José. 1997.

Fundación Omar Dengo. "Informe final de la Evaluación Formativa del Componente de Capacitación del Proyecto Informática Educativa 21 para I y II Ciclos (PIE)". Informe 13-2. Informe de investigación evaluativa. San José. 2000a.

Fundación Omar Dengo. "Informática y educación de adultos: El caso de los cursos de navegación por internet y correo electrónico para adultos mayores y ciudadanos de oro de la Fundación Omar Dengo". Informe de investigación. San José. 2000b.

Fundación Omar Dengo. "Informe de Estadísticas del Programa de Informática 
Educativa Ministerio de Educación Pública-Fundación Omar Dengo (PIE-MEP-FOD)". Informe del mes de Abril. San José. 2002.

Gómez, R.; Hunt, P. "Telecentre Evaluation: A global Perspective". PAN Global Networks, IDRC, proceedings of Conference, Far Hills Inn, Quebec. En: http://www.idrc.ac/telecentre/ evaluation/nn/00 Cov.html Consultado 3/07/01._1999.

Gómez, R.: Martínez, J. "Internet... ¿para qué? Fundación ACCESO. En: http://acceso.or.cr/PPPP Consultado el 8/11/01. 2001.

Gómez, R.; Martínez, J.; Reilly, K. "Paths beyond conectivity: Experience from Latin América and the Caribbean". United Nations Development Programme. En: http: //63.241.184.166/tcdcweb/ coop_south_journal/2001 oc/ index.html Consultado 9/1/02. 2001.

Hafkin, N.; Taggart, N. "Gender, information technology and developing countries: An analityc study". En: http: //www.usaid.gov/wid/pubs/ it01.htm Consultado 11/12/01. 2001.

Hamelink, C. "ICT's and Social Development: The Global Policy Context. UNRISD News" Number 21. Autumn/Winter, DP116. En:

http://unrisd.org/engindex/publ/ news/21eng/dp116.htm

Consultado el 23/7/01. 1999.

Herman, J. Evaluating the effects of technology in school reform. En: Means, B. (ed). "Technology and Education Reform the reality behind the promise". E.E.U.U.: Jossey-Bass Publishers. 1994.
Hilbert, M. "Latin America on its path into the digital age: Where are we? http://www.eclac.cl/publicaciones/ Desarrollo Productivo/ 5/ LCL555P/ Lc11555.pdf

Consultado 10/12/01. 2001.

Hudson, H. Telecentre evaluation: Issues and strategies. En: Colin, L.; Walker, D. (ed) (2001) "Telecentres: Case studies and key Issues". Vancouver: the Commonwealth of learning. En: http://col.org/telecentres Consultado el 7/1/02. 2001.

Honey M.; McMillan K.; Carrig, F. Perspectives on Technology and Education Research: Lessons form the Past and Present. EDC Center for Children and Technology. En: http://www.ed.gov/Technology/ TeachConf/1999/whitepapers/ paper1.html Consultado 17/10/01. 1999.

Iglesias, E. "From digital divide to digital opportunity in Latin America and the Caribbean". Inter-American Development Bank. En:

http://www.iadb.org/exr/speeches/ s191000c.htm

Consultado 20/8/01. 2000.

Ishaq, A. "La brecha informática mundial". Finanzas y Desarrollo. Vol. 38, № 3 . 2001.

Jackson, B. "Designing Projects and Project Evaluations Using The Logical Framework Approach". The World Conversation Union. En: http://www.iucn.org/themes/eval/ english/lfa.htm

Consultado 13/07/01. 2001.

Jurich, J."Computers in the classroom: How effective???". Performance 
Review Division. Canadian International Development Agency. J TechKnowlogia. Nov/Dec. 1999.

Kleiman, G. "Myths and realities about technology in K-12 schools". En:

www.edc.org/ILNT/news/issue14/ feature1.htm

Consultado 24/4/01. 2000.

Mansell, R.; When, U. (ed.) "Knowledge societies: Information society for sustainable development" Oxford University Press: London. 1998.

Martiniello, M. "Participación de los Padres en la Educación: Hacia una Taxonomía para América Latina". Development Discussion Paper № 709, June. Harvard Institute for International Development, Harvard University. 1998.

McConnell, S. "Connecting with unconnected rural stakeholders". University of Guelph: Canada.

http://www.devmedia.org/ papers.cfm?docid=117 Consultado el 7/9/01. 1988.

Menou, M. “Assesssing Methologies in Studies of the Impact of Information: A Synthesis, presented to annual meeting of the American Society of Information Science (ASIS) Pasadena CA, 26 May 1999”. En:

http://www.bellanet.org/partners/ aisi/proj/synthesis.htm Consultado el 5/12/00. 1999a.

Menou, M. "Synthesis Report-Part 1: Methodological issues. In: Connectivity in Africa: Use, Benefits and Constraints of Electronic Communications. Study carried out under the CABECA project of Padis (UN-ECA) as part of the IDRC sponsored research program on the Impact of Information on Development". En: http://www.bellanetorg/partners/ aisi/proj/conn.htm

Consultado el 5/12/00. 1999b.

Menou, M.; Potvin, J. “Toward a conceptual Framework for Learning about ICTs and Knowledge in the Process of Development: Background Document. Bellanet, Draft for Comment”. En:

http://www.bellanet.org/

ICT_res_pol/docs/

LEAP_Concept 2May2000.htm

Consultado el 11/5/01. 2000.

Menou, M. "IsICTometrics: Toward an alternative vision and process". En: http://funredes.org/olistica/ documentos/doc2 Consultado 27/6/01. 2001a.

Menou, M. "Educating citizens of the global learning society". Uned nations Development programme. En: http://63.241.184.166/ tcdcweb/coop_south_journal/ 2001_oct/index.html Consultado 9/1/02. 2001b.

Miller, D.; Slater, D. “The Internet. An Etnographic Approach. Chapter OneConclusions". Oxford, University of London. En: http://ethnonet.gold.ac.uk/ chapter.html Consultado el 9/05/01. 2000.

Morino Institute. "From access to outcomes: raising the aspirations for technology inicitiatives in low-income communities" Morino report on the digital divide. En http://morino.org/divides/ report2.htm Consultado el 18/12/01. 2001.

Morisson, D. “An outlet to growth”. World Link. Sept/Oct. 2001. 
National Research Council. "Internet Counts. Measuring the Impacts of the Internet". Office of International Affairs, National Research Council. Washington, D.C. En:

http://www.bsos.umd.ed/cidcm/ papers/ewilson/xnasresp2.htm\#exec Consultado el 20/06/01. 1998.

North Central Regional Educational Laboratory. "Learning with Technology Profile Tool". Technology in Education Consortium. U.S. En:

http://www.ncrtec.org/capacity/ profile/profwww.htm

Consultado el 14/5/01. 2000.

Lawson, T; Comber, C. "Introducing information and communication technologies into schools: The blurring of boudaries". En:

www.techsoup.org/articlepage. cfm?topicid=12\&articleid $=164$ Consultado el 28/11/01. 2000.

Lemke, C.; Martin, C.; Capella, E. "Children and computer technology". California: The David and Lucile Packard Foundation. En:

www.futurechildren.org

Consultado el 7/1/02. 2001.

OECD. "Learning to change: ICTs in schools". En:

http://electrade.gfi.fr/cgi-bin/

OECDBookShop.storefront/EN/

product/962001131P1

Consultado el 4/12/01. 2001.

PANTLEG "Revealing the soul of a project. Stories as evaluation: Towards a methodology". En: Success Stories of Rural ICTs in a Development Country. Banco de Documentos de IDRC. 2000.

PANTLEG. "Information and communication technologies for rural development and developing countries". En: http://www.bellanet.org/leap/ pantleg/index.cfm?

fuseaction=dsp_document_details\& doc_id=23\&cat_id=19

Consultado el 12/12/01. 2001.

Plaz, I. "Catálogo bibliográfico de tecnologías de la información y la comunicación para fomentar el desarrollo equitativo y sustentable en América Latina". En:

http://funredes.org/mistica/

castellano/ciberoteca/catalogo/

index.html

Consultado el 27/11/01. 1999.

Potvin, J.; Lanfranco, S.; Balson, D. "ICT Impact Assessment Framework". Bellanet, February, 2000. En: http://www.bellanet.org/ict_res_pol/ index.cfm?Fuseaction= view_article\&TheArticle $=16$ Consultado 4/07/01. 2000.

Prat, A. "Sociedad de la información y promoción de la cultura científica. Políticas públicas e indicadores de seguimiento (relatoría general)". En:

http://ricyt.edu.ar

Consultado 17/12/01. 2001.

Ritcher, C. La gestión del gasto público en educación en Centroamérica. En: “¿Hacia dónde va el gasto público en educación?. Logros y desafíos”. Serie Políticas Sociales 42, vol. III, CEPAL, ECLAC. División de Desarrollo Social, Chile. 2001.

Rohozinski, R. "Mapping Russian Cyberspace: Perspective on Democracy and the Net. Paper prepared for the UNRISD Conference on Information Technologies and Social Development", Geneva. En: http://www.unrisd.org/infotech/ conferen/russian/toc.htm Consultado 1/08/01. 1998.

Proyecto Conexiones "Modelo de Evaluación”. Informe Final. Colombia. En: 
http://conexiones.eafit.edu.co/ informes/final/Evaluacion.htm Consultado el 17/10/01. 2001.

Sagna, O. "Information Technologies and Social Development in Senegal". UNRISD News Number 23. Autumn/Winter.

ftp://ftp.unicc.org/unrisd/ outgoing/news/23e.pdf Consultado el 23/07/01. 2000.

SchoolNet S.A. "Assessment and the Evaluation of the Impact of ICT. Educator Development for ICT's: Framework, Version 1, 2". June. En: http://www.school.za/edict/edict/ assess.htm

Consultado el 29/05/01. 2000.

Sguazzi, T.; du Toit, D. "The espiral model. New options for supporting the professional development of implementers-based education. Findings from the learning for sustainability's piloting of ideas around environmental learning". Learning for Sustainbility Project. Johannesburg. 2000.

Stoll, K.; Menou, M.; Camacho, K.; Kehellady, Y. Learning about ICTs role in development: A frameworktoward a participatory, transparent and continuous process. Draft 5.2. 2002.

The Public Voice. "The Public Voice and the digital divide: a report for the DOT force". En:

www.thepublicvoice.org/dotforce/ DOT_Force_Report.pdf

Consultado el 9/1/02. 2001.
Thomas, G. "Elements of a national strategy to foster effective of techonology in elementary and secondary education". En:

www.rand.org/publications/

CT/CT145/CT145.pdf

Consultado 20/11/01. 1998.

UNRISD. "Info-Tech Project: Information Techonologies and Social Development". En:

http://www.unrisd.org/infotech/ index.htm

Consultado 31/07/01. 1998.

Van Harmelen, U. "Evaluating Change: an impact study of the Life Science Project Namibia, 1991-2000. Educational Department Rhodes University. 2001.

Wilmore, D. "Establishing a community of learners: the use of information technology (IT) as an effective learning tool in rural primary or elementary schools". En:

http://ifets.ieee.org/periodical/

vol_3_2001/

discuss_summary_april2001.html Consultado el 17/1/02. 2001.

Wood, P. "Building Gender Considerations Into ICT Evaluation Work. APC, presented at the Evaluating and Implementing ICT Strategies for the Information Age panel at the Global Knowledge Conference in Toronto, build-gender,html. Consultado el 3/07/01. 1997.

José Miguel Rodríguez García Profesor de la Escuela de Psicología de la Universidad de Costa Rica 